\title{
Genetic mapping of habitual substance use, obesity-related traits, responses to mental and physical stress, and heart rate and blood pressure measurements reveals shared genes that are overrepresented in the neural synapse
}

Majid Nikpay, Ondrej Šeda, Johanne Tremblay, Milan Petrovich, Daniel Gaudet, Theodore A Kotchen, Allen W Cowley Jr and Pavel Hamet

Hypertension Research (2012) 35, 673; doi:10.1038/hr.2012.48

Correction to: Hypertension Research (2012) 35, 585-591; doi:10.1038/hr.2011.233; published online 2 February 2012

The authors of the above article noticed an error in publication of this paper (online publication 2 February 2012) in the accompanying
Supplementary Information. In Supplementary Table S4, female and male labels were incorrectly applied. Upon publication of the article in this issue, Supplementary Information accompanying to the html version has been rectified, and now Supplementary Table S4 is presented with correct labels. 